\title{
Índices bioclimáticos, modelagem matemática e índices estatísticos para avaliação de modelos utilizados na estimativa do conforto térmico animal
}

Bioclimatic indices, mathematical modeling and statistical indices for the evaluation of models used

to estimate animal thermal comfort

Índices bioclimáticos, modelización matemática e índices estadísticos para la evaluación de los modelos utilizados en la estimación del confort térmico animal

Recebido: 25/02/2021 | Revisado: 04/03/2021 | Aceito: 04/03/2021 | Publicado: 12/03/2021

\author{
Girlene Cordeiro de Lima Santos \\ ORCID: https://orcid.org/0000-0002-6979-6321 \\ Universidade Federal da Paraíba, Brasil \\ E-mail: girlenecordeiross@gmail.com \\ Ana Maria Duarte Cabral \\ ORCID: https://orcid.org/0000-0002-6101-1104 \\ Universidade Federal Rural de Pernambuco, Brasil \\ E-mail: amdcabral@gmail.com
}

\begin{abstract}
Resumo
No julgamento do conforto ou desconforto dos animais comumente são utilizados índices bioclimáticos. Os índices mais empregados nesse tipo de avaliação são: índice de temperatura do globo negro e umidade (ITGU), índice de temperatura e umidade (ITU), e carga térmica radiante (CTR), por possuírem relação com indicadores fisiológicos e comportamentais dos animais. O ITU, não absorve efeitos da carga térmica de radiação. Por esse motivo, o ITGU e CTR, são mais indicados para sistemas de criação extensiva ou mesmo confinado, com carga térmica elevada, uma vez que, dependem da temperatura do globo negro (TGN), medida capaz de inferir sobre a homeotérmica dos animais. Todavia, a maioria das estações meteorológicas brasileiras não dispõem da TGN, o que dificulta a estimativa da quantidade de carga térmica a qual os animais ficam expostos. Portanto, o uso de variáveis meteorológicas para a elaboração de equações de estimativa da TGN, é de grande importância em ações de planejamento em diferentes sistemas de produção animal. Igualmente, os índices estatísticos empregados na avaliação do desempenho dessas equações de estimativa, são fundamentais para a sua validação. Logo, objetivou-se realizar uma revisão para elucidação de índices bioclimáticos, modelagem matemática e índices estatísticos para avaliação de modelos utilizados na estimativa do conforto térmico animal.
\end{abstract}

Palavras-chave: Estresse térmico animal; ITGU; TGN.

\begin{abstract}
In judging the comfort or discomfort of animals, bioclimatic indices are commonly used. The most used indexes in this type of evaluation are: black globe temperature and humidity index (BGHI), temperature and humidity index (THI), and radiant heat load (RHL), because they are related to physiological and behavioral indicators of the animals. The THI does not absorb the effects of the radiation heat load. For this reason, BGHI and RHL are more suitable for extensive or even confined rearing systems, with high heat load, since they depend on the black globe temperature (BGT), a measure capable of inferring on the homeothermia of the animals. However, most Brazilian weather stations do not have the BGT, which makes it difficult to estimate the amount of heat load to which the animals are exposed. Therefore, the use of meteorological variables to develop equations for estimating the BGT is of great importance in planning actions in different animal production systems. Likewise, the statistical indexes used to evaluate the performance of these estimation equations are essential for their validation. Therefore, this study aimed to perform a review to elucidate bioclimatic indexes, mathematical modeling and statistical indexes for the evaluation of models used to estimate animal thermal comfort.
\end{abstract}

Keywords: Animal heat stress; BGHI; BGT.

\section{Resumen}

Los índices bioclimáticos se utilizan habitualmente para evaluar el confort o la incomodidad de los animales. Los índices más utilizados en este tipo de evaluación son: el índice de temperatura y humedad del globo negro (WBGT), el índice de temperatura y humedad (ITH) y la carga de calor radiante (CCR), porque están relacionados con indicadores fisiológicos y de comportamiento de los animales. La ITH no absorbe los efectos de la carga térmica por radiación. Por esta razón, la WBGT y la CCR son más adecuadas para los sistemas de cría extensivos o incluso 
confinados, con alta carga térmica, ya que dependen de la temperatura del globo negro (TGN), una medida capaz de inferir sobre la homeotermia de los animales. Sin embargo, la mayoría de las estaciones meteorológicas brasileñas no disponen del TGN, lo que dificulta la estimación de la cantidad de carga térmica a la que están expuestos los animales. Por lo tanto, el uso de variables meteorológicas para la elaboración de ecuaciones de estimación del TGN es de gran importancia en la planificación de acciones en diferentes sistemas de producción animal. Asimismo, los índices estadísticos empleados en la evaluación del rendimiento de estas ecuaciones de estimación son fundamentales para su validación. Por lo tanto, se realizó una revisión de los índices bioclimáticos, la modelización matemática y los índices estadísticos para la evaluación de los modelos utilizados en la estimación del confort térmico animal.

Palabras clave: Estrés térmico animal; WBGT; TGN.

\section{Introdução}

O fator climático é uma condição marcante para a criação de animais, sobretudo, em regiões tropicais e subtropicais. Portanto, estudos bioclimáticos sempre devem ser adotados como uma ação de planejamento do pecuarista. Visto que, os animais necessitam de um ambiente com condições que favoreçam o metabolismo e homeostase do animal. Desse modo, as condições de instalação devem ser modificadas conforme o tipo de criação, o manejo adotado e clima do local (Abreu et al., 2011; Avila et al., 2013; Polli et al., 2020)

No julgamento do conforto ou desconforto dos animais pelos elementos climáticos comumente são utilizados índices, que unificam os seus efeitos e que possuem relação com indicadores fisiológicos e comportamentais dos animais (Neves et al., 2009; Avila et al., 2013; Marins et al., 2020). São bons exemplos de indicadores, o índice de temperatura do globo negro e umidade (ITGU), índice de temperatura e umidade (ITU), e carga térmica radiante (CTR) que vem sendo bastante utilizados. Tanto o ITGU como a CTR dependem da TGN, enquanto o ITU utiliza a temperatura do ar (TAR).

A TGN é medida por um termômetro de globo negro, sendo capaz de inferir sobre a homeotérmica dos animais. O globo negro é um objeto esférico de cor preta fosca que possui um termômetro no seu interior, que mede a quantidade de calor presente no ambiente. Quando instalado no local de criação dos animais é capaz de mensurar a energia oriunda da radiação solar global (RG) e demais variáveis condicionadoras do desconforto térmico, ou seja, velocidade do vento (VV), umidade relativa do ar (URA) e TAR. Todavia, a maioria das estações meteorológicas brasileiras não dispõem da TGN, o que dificulta a estimativa da quantidade de carga térmica a qual os animais ficam expostos. Logo, o uso de variáveis meteorológicas para a elaboração de equações de estimativa da TGN, é de grande importância em ações de planejamento em diferentes sistemas de produção animal.

Conforme Abreu et al. (2011), O ITU não absorve efeitos da carga térmica de radiação, sendo assim, sua utilização é recomendada para animais confinados. Em sistemas de criação extensiva ou mesmo confinado, mas com carga térmica elevada, o ITGU e CTR são mais indicados. De acordo Paulo (2009), estes últimos dois índices contemplam o efeito direto e indireto da VV, URA e TAR sobre o animal. Em ambientes semiáridos do Brasil, a alimentação animal é composta basicamente da caatinga, o que induz a criação extensiva, os rebanhos são mais expostos a intempéries climáticas. Logo, o uso do ITGU e da CTR, que dependem da TGN, acabam sendo mais recomendados para mensuração climática do ambiente.

Equações matemáticas, com base em variáveis climáticas, já foram conduzidas em alguns estudos de monitoramento bioclimático de diferentes espécies animais. A URA e TAR foram utilizadas por Turco et al. (2008), que elaboraram duas equações de estimativa da TGN, uma para o período diurno que considera URA e TAR outra para o período noturno que assume apenas TAR. Igualmente, Lourençoni et al. (2016), concluíram que é capaz estimar a TGN mediante o modelo neurofuzzy que considera dados de URA da temperatura do bulbo seco (TBS) e URA. Já Abreu et al. (2011), visando a sua estimativa em ambientes ao ar livre $\left(0 \mathrm{a} 35^{\circ} \mathrm{C}\right)$ e em ambientes cobertos correlacionaram dados de TBS com a TGN.

$\mathrm{Na}$ criação animal, os estudos bioclimáticos possibilitam a escolha da espécie que melhor se adapte a certa condição climática, por conseguinte, eleva as chances de sucesso na produção (Turco et al., 2006; Silva et al., 2008; Mendes et al., 2014). Por exemplo, animais originados da Europa se apresentam geneticamente preparados para ambientes de clima 
temperado e podem sofrer os efeitos do ambiente em países tropicais.

Costa, Costa e Araújo (2016), relatam que o estresse pelo excesso de calor intensifica a frequência reparatória, desvia energia de produção para a manutenção da temperatura corpórea e ainda promove alterações no consumo de alimentos, resultando em redução da produtividade dos animais de raças mais sensíveis. Contudo, animais mestiços e os sem raça definida (SRD) mostram uma melhor adaptação e tolerância a climas de países tropicais, por se tratarem de animais mais rústicos, como mencionado por Nascimento et al. (2014), que relatam que animais SRD não tem a sua reprodução comprometida pelo estresse calórico, quando paridas em épocas secas e manejados de forma semi-intensiva em ambientes semiáridos.

As raças exóticas mais utilizadas no Brasil, predominam nas regiões Sul e Sudeste do país. Logo, as suas explorações em outras regiões, dependem de um estudo bioclimático, que por sua vez, são conduzidas por meio de modelagens matemáticas estabelecidas a partir de variáveis climáticas (Dohler et al., 2020).

Diante do exposto, objetivou-se realizar uma revisão para elucidação de índices bioclimáticos, modelagem matemática e índices estatísticos para avaliação de modelos utilizados na estimativa do conforto térmico animal.

\section{Metodologia}

Uma busca abrangente de bibliografias sobre índices bioclimáticos, modelagem matemática e índices estatísticos para avaliação de modelos comumente utilizados na estimativa do conforto térmico animal, foi realizada por meio de uma busca criteriosa nas bases de dados: Web of Science, SciELO, repositórios institucionais e Google Scholar. A função "citado por" do Google Scholar foi incluída durante a procura dos estudos, com o intuído de aprimorar o conjunto de informações.

A pesquisa trata-se de uma revisão qualitativa, que por meio do método exploratório de informações elucida o tema do estudo (Pereira et al., 2018).

Os critérios de inclusão das bibliografias na presente pesquisa foram: I) trabalhos conduzidos entre os anos 1981 a 2020; e, II) estudos que abordassem quais quer informação relevante sobre a utilização de índices bioclimáticos, modelagem matemática e índices estatísticos para avaliação de modelos utilizados na estimativa do conforto térmico animal.

As etapas da busca das bibliografias foram conduzidas na seguinte ordem: I) busca eletrônica e acesso aos estudos com o tema de interesse da pesquisa; II) leitura minuciosa do material acessado para seleção bibliográfica; III) armazenagem das informações (tipo de estudo, método, resultados, autores e ano de publicação); e, IV) inclusão das bibliografias na análise exploratória da revisão.

\section{Revisão de Literatura}

\section{Influência do conforto térmico sobre o bem estar animal}

O estresse térmico pode ser definido como um ambiente que age na temperatura corpórea do animal, além da sua zona de adaptação. Já a faixa de temperatura em que a produção é considerada excelente e o consumo de energia para conservar a termorregulação é mínimo, denomina-se a zona termoneutra (Eloy \& Pereira, 2013).

Barros Junior et al. (2017) citam que, o animal fica em conforto térmico quando ocorre um equilíbrio constante entre a perda e o ganho de energia térmica. De acordo Oliveira et al. (2012), esse processo pode ocorrer através da passagem de calor (condução, convecção e radiação) e de umidade (evaporação via transpiração e respiração) para o ambiente.

Na Figura 1, pode ser observado que, conforme a temperatura do ambiente, existe uma faixa na qual os animais se encontram dentro da zona de conforto térmico, não havendo necessidade de intensificar a ativação dos seus mecanismos termorreguladores. Dentro desta faixa, a temperatura corporal se mantém estável, ou seja, o animal não precisa gastar mais energia para se manter dentro da sua zona de conforto térmico; energia esta que poderá ser destinada à produção de leite. Os limites dessa zona de termoregularidade são limitados pela temperatura crítica superior (TCS) e temperatura crítica inferior 
(TCI), a partir das quais o animal é acometido por estresse pelo calor e pelo frio, nessa ordem (Baêta \& Souza, 2010).

Figura 1. Zona de neutralidade.

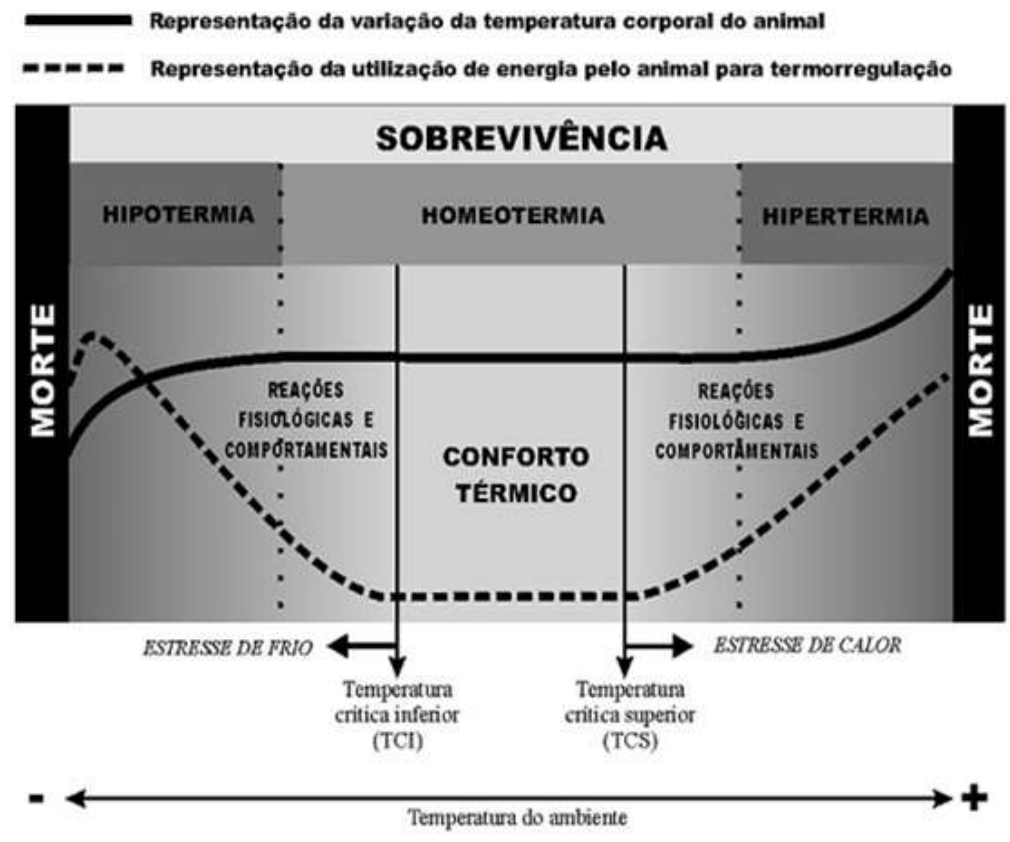

Fonte: Matarazzo (2004).

Fora de sua zona de conforto, o animal necessita ativar mais intensamente seus mecanismos fisiológicos e comportamentais para manter a temperatura corporal em equilíbrio, por meio da termólise (perder calor para o ambiente) e termogênese (produzir calor corporal). O método mais usado pelos ruminantes para trocar calor com o ambiente é a frequência respiratória (Barros Junior et al., 2017), embora este processo dependa da capacidade de saturação de URA no ambiente, o que vai facilitar ou dificultar essa troca de calor. Salles et al. (2009), concluíram que bodes Saanen em clima tropical estão susceptíveis ao estresse térmico em qualquer época do ano, mas o momento mais crítico ocorre na transição do período seco e chuvoso (primavera), quando a umidade do ar ainda é baixa e há alta temperatura, com o turno vespertino bastante desfavorável. Já Silva et al. (2019), conduziram um estudo bioclimático no nordeste brasileiro para cabras leiteiras da raça Saanen. Nesse estudo, estabeleceu-se o limite de ITGU, igual à 85, como crítico para essa raça. Nesse mesmo estudo, percebeu-se que esses animais sofrem estresse térmico durante todo o ano no estado do Maranhão, que apresentou a faixa de ITGU de 85 a 95.

No entanto, o comportamento do animal é intrínseco a cada espécie e singular para cada raça e indivíduo (Filipini, Dantas, \& Montanha, 2016). O ambiente causa desconforto e queda na produção não apenas nos animais adultos, mas durante todas as fases de desenvolvimento; por exemplo, na fase de reprodução das fêmeas pode causar alteração aguda e crônica nas concentrações plasmáticas de cortisol e hormônio tiroidianos, resultando em diminuição significativa da secreção plasmática de estradiol e elevação de progesterona, neste caso do estresse ocasionado pelo aumento da temperatura.

As respostas aos efeitos do ambiente sobre os animais podem ser avaliadas por meio de mensurações das varáveis fisiológicas: temperatura retal, frequência respiratória, temperatura superficial, taxa de sudação, frequência cardíaca, características do pelame e concentrações hormonais (Borges et al., 2016; Souza et al., 2013; Marins et al., 2020). O peso do animal é outra característica a ser usada como método indireto para avaliar estresse térmico, visto que, animais submetidos a este tipo de estresse mostram alterações no seu peso corporal (Barros Junior et al., 2017). Manteca et al. (2013), sugerem que uma única medida isolada não pode ser usada como indicador de bem estar. Eloy e Pereira (2013), avaliando a reprodução de 
caprinos machos, afirmam que o estresse térmico desencadeia falhas no progresso de reprodução através de alterações homocinéticas, que mantém a temperatura corporal, mas afeta a função reprodutiva e diminui o consumo alimentar. De acordo com estes autores, embora o estresse térmico não tenha revelado o efeito na função hormonal mostrou ter implicação sobre a maioria dos aspectos de formação de gametas e reprodução.

Portanto, a manutenção da homeostase e o controle do estresse do animal devem sempre ser almejados, de modo a incrementar a produção do animal e assegurar um produto e subproduto de melhor qualidade. O estresse ambiental desencadeia perturbações físicas nos animais como patologias, diminuição do crescimento e problemas reprodutivos (Almeida et al., 2020). Além disso, pode promover distúrbios de caráter emocionais como medo, ansiedade e agressividade (Pinheiro \& Brito, 2009), e comprometer a qualidade do produto final (Polli et al., 2020).

O clima é um fator determinante na produção, sobretudo para animais com aptidão leiteira, logo que temperaturas elevadas afetam o seu bem estar expressivamente. De acordo Broom (2011), o bem estar é um conjunto de conceitos científicos de qualidade de vida de um animal. Para Veloni et al. (2013), esses conceitos compreendem os estados naturais, mentais e físicos. Portanto, existe uma preocupação não apenas com local onde os animais são criados, mas também com o manejo, já que este pode interferir no bem estar. Por exemplo, em diferentes condições e ambientes brasileiros, estudos são conduzidos com o intuito de tornar a produção mais sustentável por meio de alternativas alimentares, utilizando variedades ou genótipos de diferentes plantas forrageiras, melhores adaptadas as condições locais, ou melhores manejadas (Santos et al., 2019; Garcia et al., 2020; Cabral et al., 2020; Oliveira, 2021; Sá, 2021); coprodutos (Santos et al., 2020); e, aditivos alimentares (Santos et al., 2020). Assim, os estudos bioclimáticos com animais em diferentes condições (ambiente, manejo, alimentação, etc.), permitem uma melhor compreensão sobre produção e a sustentabilidade da atividade. Filipini, Dantas e Montanha (2016), afirmaram que animais livres de desconforto consiste em mantê-los em ambientes com dimensionamentos corretos, além de limpos e secos.

O ambiente pode causar desconforto ao animal, de modo que o mesmo desvia a sua energia de produção para manutenção da temperatura corpórea. Borges e Rocha (2017), estudando a correlação entre as variáveis ambientais, índices bioclimáticos e parâmetros fisiológicos de caprinos criados em sistema extensivo, concluíram que a variável TAR foi a que mais influenciou os parâmetros fisiológicos dos animais.

Para análise do estresse térmico sofrido pelos animais, tanto em áreas à sombra quanto em pleno sol, podem ser usados índices de conforto (Silva et al., 2014), sendo os mais comuns o ITU, ITGU e a CTR.

\section{Índices bioclimáticos}

Esses índices são medidas bioclimatológicas essenciais para a seleção de animais mais adaptados as condições climáticas de um local ou mesmo na escolha dos melhores ambientes de criação (Barros Junior et al., 2017; Roberto et al., 2010). Os índices usualmente utilizados são o ITU, ITGU e CTR, todavia outros são citados na bibliografia, a saber.

O ITU não considera a radiação a que os amimais são expostos, ficando inviável a sua utilização em criação extensiva. Já o ITGU leva em consideração a radiação solar incidente, tornando-se mais adequado para avaliação de ambientes abertos, em razão de que, no ITGU a TAR é trocada pela TGN (Oliveira et al., 2012). De acordo com Vieira et al. (2010), o ITGU inclui direta ou indiretamente o efeito da temperatura e URA, velocidade do ar e a radiação solar incidente.

O ITGU é muito usado para vacas produtoras de leite, já para caprinos e ovinos, ainda não existe valores de ITGU padrão para a maioria das raças dessas espécies (Lima \& Barbosa Filho, 2020; Marins et al., 2020). Este índice reflete os impactos dos elementos meteorológicos, no entanto, suprime os mecanismos de termólise evaporativa dos animais (Sousa Júnior et al., 2008).

No cálculo do ITU e ITGU é usada a equação proposta por Buffington et al. (1981), citada abaixo. Porém, quando o 
intuito é calcular o ITU, T consiste na TAR, enquanto para a estimativa do ITGU, usa-se a TGN.

$\mathrm{ITGU}=\mathrm{T}+0,36 \mathrm{Tpo}+41,5$

sendo,

Tpo $=$ temperatura do ponto de orvalho $\mathrm{em}^{\circ} \mathrm{C}$.

A TGN é medida por meio de um termômetro acomodado dentro de um globo negro, que é uma esfera oca, pintada de cor preta fosca, sendo capaz de representar o calor e radiação solar recebida pelo animal em um determinado ambiente. Neste, é assumido que o corpo negro é uma construção teórica presumível, da qual sua absortividade e emissividade tornam-se máxima (Souza et al., 2002).

Por sua vez, a CTR é calculada pela equação proposta por Esmay (1969) citado por Leitão et al. (2013), que depende da VV, URA e TAR e TGN, como seguem:

$\mathrm{CTR}=\sigma(\mathrm{TRM}) 4$

em que,

CTR é em W m-2;

$\sigma=$ constate de Stefan Boltzmann, 5,67 10-8 W m-2 K-4; e,

$\mathrm{TRM}=$ temperatura radiante média em $\mathrm{K}$, em que:

$\mathrm{TRM}=100 \cdot\{2,51 \cdot \mathrm{VV} 1 / 2 .(\mathrm{TGN}-\mathrm{TAR})+(\mathrm{TGN} / 100) 4\}^{1 / 4}$

sendo,

VV é em m s1;

TGN, aqui em K; e,

$\mathrm{TAR}=$ temperatura do ar, em K.

Vários estudos confirmam a relação entre os índices bioclimáticos e variáveis fisiológicas dos animais. Façanha et al. (2012), encontraram alta relação entre o ITGU e variáveis termorreguladoras de cabras produtoras de leite no terço final da lactação. Ainda estes autores afirmam que, a homeotermia pode ser afetada pela CTR e ITGU, dado que, estes afetaram a temperatura retal e frequência respiratória do animal. Silva et al. (2017), constataram que a temperatura retal $(r=0,52)$ e a frequência respiratória $(r=0,53)$ de caprinos sem padrão racial definido, criados em sistema extensivo, apresentaram correlação com o ITGU, sinalizando que os animais ativaram seus mecanismos termorregulatórios para manter a homeostase.

De acordo o National Environment Science (2012), para bovino o ITGU de até 74 indica condição de bem estar; de 74 a 78, a condição é de cautela; de 79 a 84, a condição é perigosa e acima de 85, o estado é de emergência. Vários autores citam valores de ITGU até 74 como situação de conforto (Pereira et al., 2011; Viana, Medeiros, \& Souza, 2013). Já ITGU acima de 84 mostra situação de emergência aos animais (Medeiros et al., 2015).

Para caprinos, Souza et al. (2011), avaliando o efeito do clima sobre cabras Saanen em confinamento no Sertão Paraibano, encontraram valores de ITGU no período da tarde de 82,27 à sombra e 93,5 no ambiente ao sol. Para as condições do semiárido brasileiro, Silva et al. (2011), verificaram médias de ITGU de 80,50 e 90,51 à sombra e em pleno sol, nessa 
ordem, para caprinos mestiços F1 Saanen com Boer. Já Silva et al. (2014), avaliando a adaptação ambiental de cabras com aptidão leiteira com auxílio de precisão termográfica também no semiárido brasileiro a sombra e a pleno sol, encontraram valores de ITGU de 74,97 e 80,66 pela manhã e 79,96 e 86,90 à tarde, respectivamente. Porém, esses autores citam que apesar de os valores serem elevados, não se caracterizou como condição de perigo para as raças avaliadas, devido os caprinos terem se mantidos com a temperatura retal dentro da faixa de normalidade.

Resultados superiores foram obtidos por Pereira et al. (2011), avaliando caprinos da raça Saanen no semiárido paraibano, onde encontraram valores de ITGU a sombra e ao sol, respectivamente, pela manhã de 86,3 e 86,4 e, no turno da tarde de 89,7 e 95,1. Segundo estes autores, esta condição representou uma situação de emergência, mostrando que os animais tanto a sombra quanto a plano sol estiveram em condições de estresse térmico.

Roberto e Souza (2011), estudando parâmetros hematológicos de caprinos submetidos a diferentes níveis de suplementação no semiárido paraibano, encontraram médias de valores de ITGU de 81,35 a sombra e de 90,74 a pleno sol, valores estes que não representaram situação de risco aos animais, já que os parâmetros hematológicos se encontraram dentro do critério para a espécie caprina. Silva et al. (2010), avaliando adaptabilidade de caprinos no semiárido por meio de parâmetros fisiológicos e estruturas do tegumento, encontraram médias de ITGU de 80,11 e 92,17 a sombra e a plano sol, respectivamente.

Embora o ITU, ITGU e CTR sejam os índices mais conhecidos, outros foram criados para a avaliação do conforto térmico do ambiente para o animal. Por exemplo, o índice de conforto térmico (ICT) foi proposto por Barbosa e Silva (1995), para análise de ambientes de criação de ovinos; e, aplicado por Mendes et al. (2014), para estudo bioclimático para ovinos Dorper:

$\mathrm{ITC}=0,659 \cdot \mathrm{tAR}+0,511 \cdot \mathrm{e}+0,550 \cdot \mathrm{TGN}-0,042 \cdot \mathrm{VV}$

ICTD = TAR.0,46956+TGN.0,47194+VV.0,19221

onde,

$\mathrm{e}=$ pressão parcial do vapor d'água $(\mathrm{kPa})$.

\section{Modelagem matemática e índices estatísticos para avaliação de modelos}

$\mathrm{Na}$ modelagem matemática, tem-se a necessidade de investigação dos dados, sendo uma importante ferramenta de tomada de decisão, que se baseia na tecnologia da informação, conhecimentos matemáticos, estatísticos e do meio ambiente (Penereiro \& Ferreira, 2012; Santos et al., 2020; Dohler et al., 2020). O modelo matemático reproduz a realidade de um determinado estudo, isto é, ilustrando as suas mudanças (Sodré, 2007; Santos et al., 2020).

Os modelos matemáticos são determinados por meio do uso de regressão. Na regressão linear, simples ou múltipla, uma reta ou curva alcança ao máximo os dados associados. A análise de regressão baseia-se na produção de uma análise estatística para averiguar a presença de uma conexão entre uma variável dependente com uma ou mais variáveis independentes, ou seja, significa conseguir uma equação matemática a qual explique a alternância da variável dependente, através da variação dos níveis das variáveis independentes (Lopes, Chavarette, \& Cossi, 2017).

Para representação de evento de estudo pode ser feito um gráfico de dispersão para observação do comportamento da variável dependente $(\mathrm{Y})$ de acordo com a oscilação da variável impendente $(\mathrm{X})$. Esse comportamento pode ser linear, quadrático, cúbico ou mesmo assumir outras formas. Logo, a regressão permite obter um modelo que melhor descreva a flutuação dos dados Y em função da variável X (Peternelli, 2017). 
Na etapa de calibração, ou seja, ajuste dos parâmetros da equação, o intuito é tornar o modelo mais coeso aos dados, para diminuir a insegurança dos resultados. Para isso, a estatística permite criar estratégias para a manipulação dos dados: descrição, classificação, apresentação e conclusão dos dados (Fernandes, 1999). No entanto, é primordial conhecer o conjunto de dados (contínuo, ordinal ou nominal), sua distribuição (normal ou livre) e tipo de amostra (independente ou dependente) (Normando, Tjäderhane, \& Quintão, 2010).

Depois da calibração do modelo, procede-se a sua validação por meio de análises estatísticas. Existem alguns índices estatísticos para avaliar o desempenho desses modelos matemáticos, tai como: coeficiente de correlação de Pearson (r); coeficiente de determinação (r2); índice de concordância de Willmott, (d); erro médio de estimativa (MBE); raiz do erro quadrado médio (RMSE); e, teste t de Student.

O coeficiente de correlação de Pearson (r) é um parâmetro de combinação linear entre as variáveis (Figueiredo Filho \& Silva Júnior, 2009). O “r” mede a intensidade da conexão de duas variáveis quantitativas, podendo variar de -1 a 1 . Valores mais próximos dos extremos mostram que a combinação entre às duas variáveis é maior, sendo que mais próxima de 1 indica uma conexão linear ideal, os valores próximos de -1 apontam também uma conexão ideal, porém contrária. Já quando o valor é igual a 0 (zero) indica que não existe relação entre as variáveis (Braga et al., 2015). O r2 normaliza os valores de "r" para o intervalo de 0 a 1 , sendo que quanto mais próximo de 1 significa que o modelo proposto apresentado é apropriado para explicar o evento (Peternelli, 2004).

O índice de concordância de Willmott, representado por pela letra "d", mede o afastamento dos dados em relação à reta (1:1), ou seja, indica a veracidade dos resultados simulados em relação aos medidos. Os seus valores variam de 0 a 1 , sendo que valores próximos de 1 indicam menor amplitude de erros (Haveroth et al., 2012).

Camargo e Sentelhas (1997), sugerem uma classificação do desempenho de modelos a partir do produto entre os valores de "r" e "d", gerando um índice denominado índice de confiança "c".

O MBE indica o desempenho do modelo a longo prazo, que possui associação com o coeficiente " $r$ ", o estado de dispersão dos dados (Haveroth et al., 2012). Já o RMSE, é normalmente utilizado para medir a acurácia dos produtos numerais, apresentando valores do erro, nas mesmas proporções da variável analisada (Hallak \& Pereira Filho, 2011). Por sua vez, o teste t de Student é um teste paramétrico que indica se há diferença entre dois conjuntos de dados (Figueiró, 2014).

Pesquisas foram conduzidas nas mais diversas áreas do conhecimento com aplicação desses índices estatísticos. Abreu et al. (2011), criaram equações, que estima a TGN empregando um modelo de regressão linear. Estes autores constataram que é possível estimar a TGN a partir da TBS em ambientes cobertos com valores de 0 a $35^{\circ} \mathrm{C}$, com modelo ajustado apresentando r2 de 0,9788 e erro de predição de 3,19\%. Silva et al. (2007), por sua vez, criaram um modelo multiplicativo, capaz de estimar a URA nos estados da Bahia, Alagoas e Sergipe, utilizando distintas variáveis meteorológicas. O modelo encontrado foi capaz de explicar 81\% da variabilidade dessa variável, com valor de "r" de 0,8581, "d" de 0,9214, RMSE de 2,03\% e MBE de 0,60\%. Rosa Filho, Parisia e Soares (2018), aplicaram estes índices para a elaboração de modelos de regressão múltipla linear de estimativa da velocidade média do vento, obtendo-se r2 igual a 0,7373 e erro de 0,290. Borges Júnior et al. (2012), avaliaram métodos de cálculo da evapotranspiração de referência diária para a microrregião de Garanhuns no estado de Pernambuco. O método Hargreaves-Samani calibrado apontou melhor desempenho, com resultados para primavera-verão e outono-inverno, respectivamente, iguais a: r2 $(0,7054 ; 0,8027)$, “r” $(0,84 ; 0,90)$, “d” $(0,91 ; 0,95)$, “c” $(0,77 ; 0,86)$ e MBE $(0,40 \mathrm{~mm} ; 0,31 \mathrm{~mm})$.

Silva et al. (2019), utilizando dados meteorológicos, realizaram um estudo para estimativa da TGN em diferentes escalas de tempos. Esses autores criaram um modelo matemático, para escala diurna, utilizando apenas dados da TAR, que foi capaz de explicar $80 \%$ da TGN; um modelo múltiplo, usando dados de TAR e RG, que juntos, explicaram a TGN em 91\%; e, um modelo para escala noturna, em que, apenas a TA foi suficiente para explicar 99\% da TGN. Esses autores ainda 
desenvolveram modelos matemáticos para estimativas da TGN nas escalas diária e mensal, onde, apenas a TAR foi responsável por explicar a TGN em $97 \%$ e $98 \%$, respectivamente.

\section{Considerações Finais}

Estudos bioclimáticos devem ser adotados no julgamento do conforto ou desconforto dos animais como uma ação de planejamento do pecuarista.

Os principais índices, que possuem relação com indicadores fisiológicos e comportamentais dos animais, são: índice de temperatura do globo negro e umidade (ITGU), índice de temperatura e umidade (ITU), e carga térmica radiante (CTR).

O ITU, que depende da temperatura do ar (TAR), não absorve efeitos da carga térmica de radiação, logo é recomendada para animais confinados. Já o ITGU e CTR são mais indicados para sistemas de criação extensiva ou mesmo confinado, mas com carga térmica elevada. Por esse motivo, o ITGU e CTR, que dependem da TGN, são mais recomendados para mensuração climática do ambiente.

A TGN é medida por um termômetro de globo negro, capaz de inferir sobre a homeotérmica dos animais. O globo negro (objeto esférico de cor preta fosca e um termômetro no seu interior), mede a quantidade de calor presente no ambiente, e quando instalado no local dos animais é capaz de mensurar a energia oriunda da radiação solar global (RG), velocidade do vento (VV), umidade relativa do ar (URA) e TAR. Todavia, a maioria das estações meteorológicas brasileiras não dispõem da TGN, o que dificulta a estimativa da quantidade de carga térmica a qual os animais ficam expostos. Assim, o uso de variáveis meteorológicas para a elaboração de equações de estimativa da TGN, é de grande importância em ações de planejamento em diferentes sistemas de produção animal. Igualmente, os índices estatísticos empregados na avaliação do desempenho dessas equações de estimativa, são fundamentais para a sua validação.

Para o período diurno, a TGN pode ser estimada a partir de equações, usando a URA e TAR; apenas a TAR; ou, usando dados de TAR e RG. Para o período noturno, a TGN pode ser estimada usando apenas dados da TAR. Do mesmo modo, apenas a TAR é suficiente para explicar a TGN nas escalas diária e mensal.

Além disso, a TGN pode ser mensurada pelo modelo neuro-fuzzy, que utiliza dados de URA, temperatura do bulbo seco (TBS) e URA. Todavia, apenas os dados de TBS são suficientes para estimar a TGN em ambientes cobertos ao ar livre.

Ainda há poucos estudos conduzidos para estimativa do conforto e desconforto térmico de diferentes espécies de animais a partir de índices bioclimáticos. Logo, sugere-se a condução de pesquisas que avaliem os índices de conforto crítico para as principais espécies da cadeia produtiva pecuária, uma vez que, esse tipo de estudo, permite a escolha da espécie que melhor se adapte a certa condição climática de uma região, por conseguinte, eleva as chances de sucesso na produção.

\section{Agradecimentos}

À Coordenação de Aperfeiçoamento de Pessoal de Nível Superior (CAPES - Código Financeiro 001) pela bolsa de estudo da primeira autora.

\section{Referências}

Abreu, P. G., Abreu, V. M. N., Franciscon, L., Coldebella, A., \& do Amaral, A. G. (2011). Estimativa da temperatura de globo negro a partir da temperatura de bulbo seco. Revista Engenharia Na Agricultura, 19(6), 557-563.

Almeida, J. V. N., Marques, L. R., Marques, T. C., Guimarães, K. C., \& Leão, K. M. (2020). Influência do estresse térmico sobre os aspectos produtivos e reprodutivos de bovinos - Revisão. Research, Society and Development, 9(7), e230973837. https://doi.org/10.33448/rsd-v9i7.3837

Avila, A. D., Jácome, I. M. T. D., Faccenda, A., Panazzolo, D. M., \& Müller, E. R. (2013). Avaliação e correlação de parâmetros físiológicos e índices bioclimáticos de vacas holandês em diferentes estações. Revista Eletrônica em Gestão, Educação e Tecnologia Ambiental, 14(14), $2878-2884$.

Baeta, F. D. C., \& Souza, C. F. (2010). Ambiência em Edificações Rurais: conforto animal. (2a ed.): UFV. 
Barbosa, O. R., \& Silva, R. G. da. (1995). Índice de conforto térmico para ovinos. Boletim de Indústria Animal, 52(1), 29-35.

Barros Junior, C. P., Sousa Junior, S. C., Campelo, J., Azevedo, D., Carvalho, G., \& Sousa, P. H. A. A. de. (2017). Avaliação de parâmetros fisiológicos em diferentes raças de caprinos na Região Nordeste brasileira. Revista electrónica de Veterinária, 19(1), 1-11.

Borges Júnior, J. C., Anjos, R. J., Silva, T. J., Lima, J. R., \& Andrade, C. L. (2012). Métodos de estimativa da evapotranspiração de referência diária para a microrregião de Garanhuns, PE. Revista Brasileira de Engenharia Agrícola e Ambiental, 16(4), 380-390.

Borges, L. S., \& Rocha, F. S. B. (2017). Variáveis fisiológicas de caprinos sem padrão racial definido criados em sistema extensivo. Revista Electrónica de Veterinaria, 18(11), 1-14.

Borges, L. S., Evangelista, A. F., Barros Junior, C. P., Silva, A. L., \& Andrade, T. V. (2016). O ambiente semiárido brasileiro influencia as respostas fisiológicas de caprinos. Journal of Animal Behaviour and Biometeorology. Mossoró, 4, 17-21.

Braga, D. C., Bortolini, S. M., Ferreira, R. L., Almeida, R. de., Schlegel, C., Casara, M. E. M. (2015). Consumo alimentar associado ao câncer colorretal - um estudo ecológico. GED gastroenterol. endosc. dig.,34(2), 60-65.

Broom, D. M. (2011). Animal welfare: concepts, study methods and indicators. Revista Colombiana de Ciencias Pecuarias, $24(3), 306-321$.

Buffington, D. E., Collazo-Arocho, A., Canton, G. H., Pitt, D., Thatcher, W. W., \& Collier, R. J. (1981). Black globe-humidity index (BGHI) as comfort equation for dairy cows. Transactions of the ASAE, 24(3), 711-0714.

Cabral, A. M. D., Carvalho, F. F. R., Santos, G. C. de L., Ferreira, J. C., Silva, M. J. M. S., Santos, G. R. A., \& Melo, N. D. (2020). Use of sugar cane to feed lactating dairy goats. Arquivo Brasileiro de Medicina Veterinária e Zootecnia, 72(6), 2297-2307.

Camargo, A. D., \& Sentelhas, P. C. (1997). Avaliação do desempenho de diferentes métodos de estimativa da evapotranspiração potencial no Estado de São Paulo, Brasil. Revista Brasileira de agrometeorologia, 5(1), 89-97.

Costa, A. N. L. da., Costa, A. A. da., Araújo, É. P de. (2016). Efeitos do estresse térmico na reprodução de fêmeas bovinas. Revista Brasileira de Reprodução Animal, 40(4),123-125.

Dohler, R. E., Zanetti, S. S., Cecílio, R. A., Pezzopane, J. E. M., \& Xavier, A. C. (2020). Aplicação de diferentes métodos de calibração do modelo de Hargreaves-Samani no Sudeste do Brasil. Research, Society and Development, 9(8), e368984811. https://doi.org/10.33448/rsd-v9i8.4811

Eloy, A. M., \& Pereira, E. P. (2013). Estresse na reprodução de caprinos machos. Revista Brasileira de Reprodução Animal, $37(2), 156-163$.

Eustáquio Filho, A., Teodoro, S. M., Chaves, M. A., Santos, P. E. F. D., Silva, M. W. R. D., Murta, R. M., \& Souza, L. E. B. D. (2011). Zona de conforto térmico de ovinos da raça Santa Inês com base nas respostas fisiológicas. Revista Brasileira de Zootecnia, 40(8), $1807-1814$.

Façanha, D. A., Vasconcelos, A. M., Lima, F. R., Eloy, A. M. X., Oliveira, A. L. de., Guilhermino, M. M., \& Landim, A. V. (2012). Características termorreguladoras e desempenho de cabras leiteiras no terço inicial da lactação em clima tropical. Revista Portuguesa de Ciências Veterinarias, 111(583584), 151-156.

Fernandes, M. G. (1999). Estatística aplicada. Braga: Universidade do Minho.

Figueiredo Filho, D. B., \& Silva Júnior, J. A. da. (2009). Desvendando os Mistérios do Coeficiente de Correlação de Pearson: o Retorno. Revista Política Hoje, 8(1), p. 66 .

Figueiró, R. (2014). Manual prático de Bioestatística Computacional. Volta Redonda, RJ: Centro Universitário de Volta Redonda - UniFOA Campus Três Poços.

Filipini, B., Dantas, A., \& Montanha, A. A. (2016). Bem-estar e comportamento de ovinos em sistema intensivo. Enciclopédia Biosfera, 13(24).

Garcia, P. H. D. M. (2020). Valor nutricional da silagem de genótipos de milho e sorgo cultivados em duas densidades de semeadura. In: Nutrição e Produção Animal, (1a ed.), Org. Carlos Alexandre Oelke. Guarujá, SP: Científica Digital. 335- 359.

Hallak, R., \& Pereira Filho, A. J. (2011). Metodologia para análise de desempenho de simulações de sistemas convectivos na região metropolitana de São Paulo com o modelo ARPS: sensibilidade a variações com os esquemas de advecção e assimilação de dados. Revista Brasileira de Meteorologia, 26(4), 591608 .

Haveroth, R., Pitz, J. W., Eli, K., Neves, L. D. O., \& Fernandes, E. (2012). Modelagem para a estimativa da umidade relativa do ar para a cidade de Ituporanga/SC. Enciclopédia Biosfera, 8(15), 2474-2482.

Leitão, M. M., Oliveira, G. M. D., Almeida, A. C. D., \& Sousa, P. H. de. (2013). Conforto e estresse térmico em ovinos no Norte da Bahia. Revista Brasileira de Engenharia Agrícola e Ambiental, 17(12), 1355-1360.

Lima, L. R., \& Barbosa Filho, J. A. D. (2020). Impact of pre-slaughter management on the welfare of goats and sheep. Journal of Animal Behaviour and Biometeorology, 1(2), 52-60.

Lopes, M. L. M., Chavarette, F. R., \& Cossi, A. M. (2017). Avaliação do modelo de regressão linear múltipla e redes neurais artificiais na previsão do ganho de massa em animais. Revista Brasileira de Engenharia de Biossistemas, 11(1), 01-17.

Lourençoni, D., Pena, M. R. D. S., Yanag Junior, T., Moraes, K. C. R., \& Costa, L. P. da. (2016). Modelagem neuro-fuzzy para estimar a temperatura de globo negro. In: CONGRESSO TÉCNICO CIENTÍFICO DA ENGENHARIA E DA AGRONOMIA, 2016, Foz do Iguaçu. Anais...Foz do Iguaçu, 2016.

Lucena, L. F. D. A., Furtado, D. A., Nascimento, J. W., Medeiros, A. N. D., \& Souza, B. B. D. (2013). Respostas fisiológicas de caprinos nativos mantidos em temperatura termoneutra e em estresse térmico. Revista Brasileira de Engenharia Agrícola e Ambiental, 17(6), 672-679. 
Manteca, X., Silva, C. A., Bridi, A. M., \& Dias, C. P. (2013). Bem-estar animal: conceitos e formas práticas de avaliação dos sistemas de produção de suínos. Semina: Ciências Agrárias, 34(2), 4213-4229.

Marins, T. N., Almeida, I. G. B., Lôbo, B. V., Pessoa, C. M. B., Teixeira, R. C., Alves, B. G., \& Gambarini, M. L. (2020). Índices de estresse e conforto térmico associados aos parâmetros fisiológicos e perfil energético em vacas Girolando criadas a pasto na savana tropical. Research, Society and Development, 9(7), e111973672. https://doi.org/10.33448/rsd-v9i7.3672

Matarazzo, S. V. (2004). 143 f. Eficiência do sistema de resfriamento adiabático evaporativo em confinamento do tipo Freestall para vacas em lactação. 143 f. Universidade de São Tese (Doutorado em Agronomia) - Universidade de São Paulo, Escola Superior de Agricultura Luiz de Queiroz, Piracicaba.

Medeiros, L. F. D., Rodrigues, V. C., Vieira, D. H., Souza, S. L. G. de., Cabral Neto, O., Figueiredo, N. de., \& Violento, C. B. (2015). Reações fisiológicas de cabras em diferentes ambientes e coeficiente de tolerância ao calor em cabritos. Brazilian Journal of Veterinary Medicine, 37(4), $286-296$.

Mendes, A. M. D. P., Azevedo, M. D., Lopes, P. M. O., \& Moura, G. B. D. A. (2014). Zoneamento bioclimático para a raça ovina Dorper no Estado de Pernambuco. Pesquisa Agropecuária Brasileira, 49(12), 986-993.

Nascimento, H. V. C., Oliveira, F. A., Turco, S. H. N., Cordeiro, M. F., Lopes Júnior, E. D. (2014). Fatores meteorológicos sobre a atividade reprodutiva de cabras leiteiras na época seca do semiárido pernambucano. Revista Brasileira de Engenharia Agrícola e Ambiental, 18(5), 539-544.

National Environment Science. (2012). 3(1), 42-50.

Neves, M. L. M. W., Azevedo, M., Costa, L. A. B., Guim, A., Leite, A. M., \& Chagas, J. C. (2009). Níveis críticos do índice de conforto térmico para ovinos da raça Santa Inês criados a pasto no agreste do Estado de Pernambuco. Acta scientiarum. Animal sciences, 31(2), 169-175.

Normando, D., Tjäderhane, L., \& Quintão, C. C. A. (2010). A escolha do teste estatístico-um tutorial em forma de apresentação em PowerPoint. Dental Press Journal of Orthodontics, 15(1), 101-106.

Oliveira, F. de S., Fernandes Neto, V. de P., Nascimento, M. N., Cardoso, F. S., \& Costa, A. P. R. (2012). Efeito do estresse térmico sobre os parâmetros fisiológicos e bioquímicos de ovinos criados em clima tropical. PUBVET, 6(16), 1-24, Art-1357.

Oliveira, T. C. de. (2021). A cultura do sorgo sacarino: revisão. Research, Society and Development, 10(2), e28610212755. https://doi.org/10.33448/rsdv10i2.12755

Paulo, J. L. D. A. Índices de conforto térmico para caprinos das raças moxotó e canindé em confinamento no semi-árido paraibano. 82 f. Dissertação (Mestrado em Zootecnia) - Universidade Federal da Paraíba. Programa de Pós-graduação em Zootecnia, Areia.

Penereiro, J. C., \& Ferreira, D. H. L. (2012). A modelagem matemática aplicada às questões ambientais: uma abordagem didática. Millenium, 42(1), 27-47.

Pereira, A. S. et al. (2018). Metodologia da pesquisa científica. UFSM.

Pereira, G. M., Souza, B. B. de., Silva, A. M. de A., Roberto, J. V. B., \& Silva, C. M. B. A. de. (2011). Avaliação do comportamento fisiológico de caprinos da raça Saanen no semiárido paraibano. Revista verde de agroecologia e desenvolvimento sustentável, 6(1), 21

Peternelli, L. A. (2017). Correlação Amostral, Regressão linear Simples e Regressão Linear Múltipla. Recuperado de http://www.dpi.ufv.br/ peternelli/inf162.www.16032004/materiais/CAPITULO9.pdf

Polli, V. A., Costa, P. T., Garcia, J. A. B., Restle, J., Dutra, M. M. M., \& Vaz, R. Z. (2020). Estresse térmico e qualidade da carne ovina - uma revisão. Research, Society and Development, 9(9), e595997578. https://doi.org/10.33448/rsd-v9i9.7578

Pinheiro, A. A., \& Brito, I. F. de. (2009). Online Bem-estar e Produção Animal. Sobral, CE.

Roberto, J. V. B., Souza, B. B. de., Silva, A. L. N. da., Justiniano, S. V., \& Freitas, M. M. S. (2010). Parâmetros hematológicos de caprinos de corte submetidos a diferentes níveis de suplementação no semi-árido paraibano. Revista Caatinga, 23(1), 127-132.

Roberto, J. V. B., \& Souza, B. B. de. (2011). Fatores ambientais, nutricionais e de manejo e índices de conforto térmico na produção de ruminantes no semiárido. Revista verde de agroecologia e desenvolvimento sustentável, 6(2), 8-13.

Rosa Filho, C. de D., Parisia, S. A., \& Soares, W. de A. (2018). Avaliação da distribuição estatística e elaboração de modelo de regressão múltipla linear da velocidade média do vento. Journal of Environmental Analysis and Progress, 3(1), 24-36.

Sá, M. K. N. de., Andrade, A. P. de., Magalhães, A. L. R., Valença, R. de L., Campos, F. S., Araújo, F. dos S., \& Araújo, G. G. L. de. (2021). Silagem de palma forrageira com Gliricidia Sepium: alternativa alimentar para o Semiárido. Research, Society and Development, 10(2), e27210212473. https://doi.org/10.33448/rsd-v10i2.12473

Salles, M. G. F., Souza, C. E. A., Rondina, D., Moura, A. A. A., \& Araùjo, A. A. (2009). Respostas fisiológicas ao estresse térmico de bodes Saanen em clima tropical. Ciência Animal, 19(1), 19-28.

Santos, G. C. de L., Garcia, P. H. de M., Santos, D. G., Gonzaga Neto, S., \& Duarte, A. M. C. (2020). Modelagem na nutrição de não ruminantes: aspectos gerais, dinâmica de nutrientes, limitações e sistemas de serviços. Brazilian Journal of Animal and Environmental, 3(1), 114-132.

Santos, G. C. L., Garcia, P. H. de M., Viana, T. B. L., Borges, P. F., Araujo, L. S., \& Gonzaga Neto, S. (2020). Growth and efficiency of sorghum water use under different continuous water regimes. Water stress in sorghum crop. Archivos de zootecnia, 69(265), 164-171.

Santos, G. C. de L., Gonzaga Neto, S., Bezerra, L. R., \& Medeiros, A. N. de. (2020). Uso de tortas na alimentação de vacas leiteiras: uma revisão. Brazilian Journal of Animal and Environmental Research, 3(1), 89-113. 
Santos, G. C. de L., Oliveira, J. S., Gonzaga Neto, S., \& Duarte, A. M. C. (2020). Uma revisão de quitosana: polímero que melhora a eficiência do rebanho e a sustentabilidade do sistema de produção. Brazilian Journal of Animal and Environmental Research, 3(1), 73-88.

Silva, C. M. B. de A., Souza, B. B., Brandão, P. A., Marinho, P. V. T., \& Benício, T. M. A. (2011). Efeito das condições climáticas do semiárido sobre o comportamento fisiológico de caprinos mestiços F1 Saanen x Boer. Revista Caatinga, 24(4), 195-199.

Silva, E. M. N., Souza, B. B., Silva, G. A., Alcântara, M. D. B., Cunha, M. G. G., \& Marques, B. A. A. (2014). Avaliação da adaptabilidade de caprinos leiteiros no semiárido brasileiro com auxílio da termografia infravermelha. Journal Animal Behaviour Biometeorology, 2(3), 95-101.

Silva, P. T., Pádua, R., Reis, J., Amaral, A., Silva, A., \& Carvalho, R. (2017). FE Avaliação da adaptação de bovinos da raça Senepol nas condições climáticas do cerrado goiano. Revista Espacios, 38(26), 1-12.

Silva, T. D., Zolnier, S., Moura, M. D., \& Sediyama, G. C. (2007). Estimativa e espacialização da umidade relativa do ar para os estados de Alagoas, Bahia e Sergipe. Revista Brasileira de Agrometeorologia, 15(1), 14-28.

Silva, T. G. F., Moura, M. S. B., Sá, I. I. S., Zolnier, S., Turco. S. H. N., \& Souza, L. S. B. de. (2010). Cenários de mudanças climáticas e seus impactos na produção leiteira em estados nordestinos. Revista Brasileira de Engenharia Agricola e Ambiental, 14(8), 863-870.

Silva, T. G. F., Santos, G. C. de L., Duarte, A. M. C., Turco, S. H. N., Cruz Neto, J. F., Jardim, A. M. D. R. F., \& Santos, T. S. dos. (2019). Black globe temperature from meteorological data and a bioclimatic analysis of the Brazilian Northeast for Saanen goats. Journal of thermal biology, 85, 102408.

Silva, T. G. F., Turco, S. H. N., Zolnier, S., Soelma, M., Moura, B., \& Sá, I. I. S. (2008). Variação regional do declínio na produção de leite durante o verão no estado de pernambuco. Engenharia na Agricultura, 16(1), 109-123.

Sodré, U. (2007). Modelos matemáticos. Londrina: UEL.

Sousa Júnior, S. C., Morais, D. A. E. F., Vasconcelos, Â. M., Nery, K. M., Morais, J. H. G., \& Guilhermino, M. M. (2008). Características termorreguladoras de caprinos, oivinos e bovinos em diferentes épocas do ano em Região Semi-Árida. In: Embrapa Caprinos e Ovinos-Artigo em anais de congresso (ALICE). Revista Científica de Produção Animal, 10(2), 127-137.

Souza, B. B., Assis, D. Y. C., Silva Neto, F. L., Roberto, J. V. B., \& Marques, B. A. de A. (2011). Efeito do clima e da dieta sobre os parâmetros fisiológicos e hematológicos de cabras da raça Saanen em confinamento no sertão paraibano. Revista verde de agroecologia e desenvolvimento sustentável, 6(1), 77-82.

Souza, C. D. F., Tinôco, I. D. F., Baêta, F. D. C., Ferreira, W. P. M., \& Silva, R. D. (2002). Avaliação de materiais alternativos para confecção do termômetro de globo. Ciência e Agrotecnologia, 26(1), 157-164.

Souza, R. B., Nascimento, M. R. B. de M., \& Igarasi, M. S. (2013). Características termorreguladoras de caprinos em ambiente tropical: revisão. $P U B V E T, 7(6), 1-8$.

Turco, S. H., Silva, T. G. F.., Santos, L. F. dos., Ribeiro, P. H., Araújo, G. G. de., Holanda Junior, E. V., \& Aguiar, M. A. (2006). Zoneamento bioclimático para vacas leiteiras no estado da Bahia. Engenharia Agrícola, 26(1), 20-27.

Turco, S. N., Silva, T. G. F. da., Oliveira, G. M. de., Leitão, M. R., Moura, M. B. de., Pinheiro, C., \& Padilha, C. V. da S. (2009). Estimating black globe temperature based on meteorological data. In: INTERNATIONAL LIVESTOCK ENVIRONMENT SYMPOSIUM, 8, 2008, Foz do Iguaçu. Anais... Livestock Environments, Proceedings. American Society of Agricultural and Biological Engineers. Foz do Iguaçu, USA: ASABE, 2008. p. 122

Veloni, M. L., Prado, P. L., Arssuffi, B. M., Ballestero, M. C. M., Oliveira, M. G., Abreu, P. B., \& Oliveira, L. G. (2013). Bem-estar animal aplicado nas criações de suínos e suas implicações na saúde dos rebanhos. Revista Científica Eletrônica de Medicina Veterinária, 21(1), 1-21.

Viana, M. P., Medeiros, A. da R., \& Souza, B. B. de. (2013). Efeitos do estresse térmico sobre a fisiologia, produção e reprodução de caprinos. Agropecuária Científica no Semiárido, 9(4), 01-08.

Vieira, R. D. F. N., Oliveira, K. S., de Paula, S. M., \& Martins, J. R. (2010). Índices de conforto na avaliação do bem estar animal. Nucleus Animalium, 2(1), $63-70$. 